\title{
VINCENT BERTHELIER
}

Université de la Sorbonne

\section{Stylistique du passéisme}

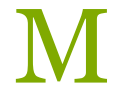

on article se propose d'aborder la question du retour, non à travers des thèmes, mais à travers le prisme du style. En lisant deux auteurs qui ont en commun d'amorcer, au moyen de la littérature, un retour à une époque qu'on pourrait dire prémoderne ou précapitaliste, je m'interrogerai sur la possibilité de dégager des traits similaires, une unité de ton qui les rassemblerait, par exemple, autour d'une certaine attitude désabusée devant le présent et d'une certaine inquiétude face à la modernité.

Les auteurs que j'ai choisi de présenter sont clairement des auteurs confidentiels à l'heure actuelle, mais leur pratique d'écriture m'a semblé constituer une démarche significative de mise en forme de ce retour, et même plus précisément du passéisme désabusé qui les caractérise. II s'agit de Baudouin de Bodinat, auteur de $L a$ vie sur Terre (premier tome paru en 1996), et d'Au fond de la couche gazeuse (2015), et de Richard Millet, romancier et pamphlétaire. On trouve chez eux un même rejet global de la société moderne, mais donnant lieu chez Millet à une déploration réactionnaire de la décadence et du progressisme " bien-pensant ", chez Bodinat à la vitupération de la technicisation du monde et de ses divers avatars.

À partir de tels textes, produits dans les mêmes années et à partir d'un constat comparable, quoique se donnant des horizons divergents, je me demanderai si on peut dégager un objet, qui soit comme un style du pass é i s m e, un style propre à un auteur qui regarderait en arrière. Pour cela j'examinerai différentes approches sty- 
listiques que l'on peut mettre en œuvre, et leur pertinence respective pour le corpus que j'ai désigné. Avant toutefois de commencer, on notera la difficulté particulière de l'objet, le retour, ou, comme j'ai choisi de le reformuler au prix d'une simplification et d'un changement d'orientation, le passéisme. En effet ce dont on traite est une attitude conjointement idéologique, voire politique, et affective. Affective en ce que le retour fait écho à des affects individuels comme la nostalgie ou la mélancolie. Idéologique, en ce que la question du retour se pose depuis une situation matérielle collective, la modernité, le caractère mondial du capitalisme industriel, et des réactions s'opposant à lui : anti-modernisme, critique du progrès, regret des formes de vie passées. Or cette difficile articulation du collectif et de l'individuel me semble ici analogue à celle qui pose problème à la stylistique, le style étant tantôt défini comme l'expression d'une singularité affective, comme le propre d'un auteur, et tantôt comme un ensemble de faits textuels repérables d'une œuvre à une autre, et susceptibles d'être réunis dans une catégorie commune (style pamphlétaire, style NRF, écriture blanche, etc.).

\section{Rhétorique}

Dans Les Antimodernes, A. Compagnon rassemble les styles antimodernes sous l'étiquette de la vitupération (dont les signes extérieurs sont l'emploi de mots-valises, la juxtaposition avec contraste de registre, le paradoxe, l'oxymore et l'antithèse, la véhémence, le style prophétique par la violence des images, à la manière d'Isaïe - l'ampleur des périodes $\left.{ }^{1}\right)$. En somme, un style qui transvaserait dans l'écriture la vitalité perdue dans l'Histoire. Compagnon caractérise donc leur style en se référant à un g e $\mathrm{n}$ r e rhétorique, le discours épidictique, qui a pour fonction de faire l'éloge ou le blâme (uituperatio). Le style, à partir de la

${ }^{1}$ A. Compagnon, Les Antimodernes : de Joseph de Maistre à Roland Barthes, Paris, Gallimard, 2005. 
tradition rhétorique, serait essentiellement un choix au sein d'un nombre fini de possibilités, plus ou moins équivalentes du point de vue du sens.

Dans la tradition rhétorique, ce qu'on désigne par st yle, ce sont des niveaux de discours, le choix d'un certain lexique, de certaines figures. Le style est élevé, médiocre ou humble, selon que les mots choisis sont plus ou moins nobles, le choix du style s'adaptant à l'objet traité.

Si l'on essaie dans un premier temps de caractériser les styles de Bodinat ou de Millet dans une perspective rhétorique, on est intuitivement conduit à les placer du côté du style élevé. Mais il reste à savoir ce que cette catégorisation un peu intuitive signifie. Le style passéiste pourrait en effet consister à revenir à une manière d'écrire située dans le passé : à une écriture de facture classique, voire archaïque. Dans le cas de Bodinat, ce modèle stylistique serait à chercher du côté de la Bible. En effet, un des faits textuels les plus saillants de ses essais est l'emploi de la conjonction et à l'initiale des phrases, qui revient à peu près à chaque nouveau paragraphe :

Et à cet instant de triomphe sur sa lâcheté et son abattement de tout à l'heure on voit sur le visage de l'individu à la peau en effet bien lustrée par les crèmes de soin e t les compléments nutritionnels, s'esquisser l'ombre d'un sourire. ${ }^{2}$

La conjonction placée à l'initiale se trouve dans la Genèse et dans l'Évangile de Luc, généralement répétée à chaque phrase, et elle a servi, spécifiquement en tête d'alinéa, de marqueur stylistique à d'autres écrivains chrétiens comme Lamennais ou Claudel ${ }^{3}$. Cette " respiration

${ }^{2} \mathrm{~B}$. de Bodinat, La vie sur Terre : réflexions sur le peu d'avenir que contient le temps où nous sommes, Paris, Éditions de l'Encyclopédie des Nuisances, 2008, p. 113.

${ }^{3} \mathrm{G}$. Antoine, La coordination en français, Paris, Éditions D’Artrey, 1958, p. 940 et G. Antoine, Les Cinq Grandes Odes de Claudel ou La poésie de la répétition, Paris, Minard, 1959, p. 36-38. On peut penser également au début d'Une Saison en enfer : "Et je l'ai trouvée amère. - Et je l'ai injuriée " (A. Rimbaud, Une saison en enfer, Alliance typographique, Bruxelles, 1873, 
biblique ", déjà remarquée par les critiques, comme Sébastien Lapaque ${ }^{4}$, émane aussi de l'anaphore des présentatifs, également en début d'alinéa : "Voici encore ce que j'ai vu ", "Voici ce que j'ai pensé d'autre " $^{5}$. Un genre de formule qui se trouve plutôt cette fois dans l'Ecclésiaste $(3,16$ et 5,17$)$.

Ce tic stylistique est encore plus récurrent dans son deuxième grand essai, Au fond de la couche gazeuse, et rendu plus visible par l'usage de l'esperluette, employée seulement pour les " et " situés en tête de phrase et de paragraphe : « \& c'est la raison que, par nature d'affinité, qui ignore la nostalgie, qui ne se cherche plus dans luimême et dans le monde, ne peut non plus connaître l'amour, ni même avoir quelque idée de quoi il s'agit $»^{6}$. La typographie elle-même contribue à cet effet archaïsant, l'esperluette n'étant plus guère utilisée que dans les sigles commerciaux. Cependant l'emploi de l'esperluette un iqu e me $\mathrm{nt}$ en tête d'alinéa trahit la volonté de produire un effet de style visible et lisible (alors que la typographie ancienne utilisait cette ligature systématiquement). Enfin, on serait même tenté de voir, jusque dans la matérialité du livre, un choix passéiste, La vie sur Terre ayant été, comme tout le catalogue de l'Encyclopédie des nuisances, composé sur linotype (avec des caractères en plomb, dans l'une des dernières imprimeries n'étant pas passée à l'offset).

Si l'on revient sur l'extrait cité, on remarque de nombreuses formes en qu-, qui donnent ce grain rugueux à la

p. 1). Dans tous les cas, G. Antoine considère que cet « et » n'est pas conforme à notre conscience linguistique. Ce serait donc nettement un " écart » expressif.

${ }^{4}$ S. Lapaque, Autrement et encore, Paris, Actes Sud, 2013, p. 127.

${ }^{5}$ B. de Bodinat, La vie sur Terre, op. cit., p. 57.

${ }^{6}$ B. de Bodinat, Au fond de la couche gazeuse : 2011-2015, Paris, Fario, 2015, p. 120. Cet essai est composé en grande partie d'articles publiés dans la revue Fario. L'emploi de l'esperluette date des premières publications dans cette revue, comme on peut le voir avec le texte " La grande féerie ", publié dans la dernière édition de La vie sur Terre, mais prépublié dans le numéro 4 de Fario. 
phrase. Cette phrase compose une maxime, comme on en écrivait au Grand Siècle (gardons en tête toutefois qu'il s'agit d'un genre vivant jusqu'à la première moitié du XX siècle, même s'il garde une dignité classique). Elle repose sur des procédés de généralisation qu'on retrouve également dans les proverbes, comme le présent gnomique bien sûr, mais aussi la relative substantive : " qui ignore la nostalgie, qui ne se cherche plus dans lui-même et dans le monde, ne peut non plus connaître l'amour ${ }^{7}$ (au lieu de celui qui ou de quiconque). Mais les choix stylistiques ne dépendent pas seulement de la visée générale du discours. Ainsi le qu e relateur omni-fonction (" c'est la raison que " au lieu de "c'est la raison pour laquelle ") ou le pronom interrogatif indirect " quelque idée de quoi il s'agit ", à la place du tour relatif plus fréquent " de ce dont il s'agit ". Les choix stylistiques que l'on peut isoler (en les comparant à d'autres formulations possibles de sens plus ou moins équivalent) renvoient à des corpus ou à des genres historiquement datés, vieillis (ce sont les stylèmes de la Bible, des maximes ou des proverbes), et l'effet est renforcé par des locutions archaïsantes ${ }^{8}$.

En ce qui concerne Richard Millet, les choix stylistiques vont aussi dans le sens d'un retour en arrière, mais selon des modalités différentes. Ces choix sont en effet explicités dans ses essais (quand ce n'est pas sous forme de mise en abyme méta-discursive comme dans son récit $\operatorname{Tarnac}^{9}$ ), où il se réfère notamment à Bossuet, Saint-

\footnotetext{
7 Ibidem.

8 Je ne reviendrai pas sur l'association complexe entre archaïsme et classicisme, qui conduit pour certaines époques à exclure du style classique les archaïsmes, en ce qu'ils réduisent la lisibilité du texte (voir L. Himy-Pieri, S. Macé (éd.), Stylistique de l'archaïsme : Colloque de Cerisy, Bordeaux, Presses universitaires de Bordeaux, 2010). On pourrait néanmoins se demander, pour la période contemporaine, si l'archaïsme n'est pas redevenu un marqueur de style classique, la catégorie " style classique " ayant perdu sa centralité et sa vocation universelle.

${ }^{9}$ " Pascal Bugeaud [...] aux phrases plus longues qu'un vendredi saint " (R. Millet, Tarnac, Paris, Gallimard, 2010, p. 14). Pascal Bugeaud est le double littéraire de Millet, le héros et narrateur de Ma vie parmi les ombres.
} 
Simon, Chateaubriand, Proust et Claude Simon, auteurs pratiquant tous la phrase longue. Bien évidemment il y a de longues phrases chez ces auteurs, néanmoins leurs structures sont nettement différentes, selon qu'il s'agit, chez Bossuet, de périodes oratoires construites autour de balancements, avec protase et apodose, de phrases allongées par des parenthèses et des incidentes comme chez Proust, ou par les épanorthoses presque maniaques de Claude Simon. En somme, chez Millet, dont le style se rapprocherait plus volontiers d'une version assagie de l'écriture simonienne ${ }^{10}$, comme en conviennent plusieurs critiques, la lecture est orientée par une grille stylistique présente dans le corps du texte (y compris dans ses fictions ${ }^{11}$ ), et cette grille est marquée par son anti-historicisme, écrasant les différences entre siècles et contextes sous une même pratique d'écriture. Le retrait de l'histoire est d'ailleurs assimilable à la position de la littérature ellemême dans la vision de Millet. Le choix stylistique le plus saillant de Millet épouse donc une perspective passéiste par le dispositif méta-discursif mis en avant (et en général, par la cohésion entre ses essais et ses fictions), qui associe la phrase longue à un classicisme français en voie de disparition.

Elle n'entendit pas, se revoyant probablement, les soirs où il y avait du monde, la gaule à l'épaule, derrière les cochons qu'elle faisait descendre par une sente rude vers la buige qui poussait au bas du pré, battant le sol pour chasser les serpents et demeurant là jusqu'à ce que ce fût terminé, c'est-à-dire à la fin de l'après-midi ou, si c'était un dîner, à nuit tombée, alors que le pays était vaincu, humilié, occupé, que ces Barbatte-là fai-

${ }_{10}$ Notamment du fait que ses phrases s'allongent chez lui par la droite, par adjonction de subordonnées ou d'apposition, et non, comme chez Proust, par le milieu. L'influence proustienne est essentiellement thématique ou plutôt philosophique, l'obsession du Temps en est un exemple. Le fossé qui sépare Millet de Bossuet a bien été démontré par l'article de Laurence Plazenet "Voix de l'ombre et du néant ", [dans :] J.-Y. Laurichesse (éd.), Littératures, Toulouse, Presses universitaires du Mirail, 2011, vol. 63, p. 38.

11 Une des raisons qui m'ont poussé à faire partiellement abstraction des frontières génériques. 
saient comme si rien n'était changé, que l'aîné des frères, plutôt que de trafiquer son bourgogne avec l'occupant, avait gagné Londres, et que les godelureaux continuaient de venir ripailler au Montheix, sans rien voir ni entendre $[\ldots] .^{12}$

Ce n'est pas là le seul aspect du style de Millet. Si l'on poursuit l'examen d'un point de vue rhétorique, il semblerait que Millet ait opté pour le style élevé (gravis), avec cependant une curiosité rhétorique : l'inadéquation entre ce style élevé et les sujets abordés, qui sont humbles ou vils $^{13}$. Millet parle de paysans, d'aliments, du corps et de ses sécrétions. Tous ces référents sont mis à distance, voire parfois anoblis. Cela se fait par un lexique plus savant, la " sentine ", les " brages empouacrées » ${ }^{14}$, formule qui combine un terme occitan (les " braies ") avec un participe précieux qu'on ne trouve guère que chez Huysmans ou Jean Lorrain. On peut également comparer la formule de Lauve le pur, " la grande fraternité de l'excrément ${ }^{15}$, au " grand communisme du caca » célinien : réécriture symétrique, mais pour laquelle Millet a choisi des termes plus abstraits, plus éloignés des enjeux politiques immédiats, et de registre neutre. Qu'il s'agisse de l'emploi d'un lexique savant trop recherché (son emploi systématique du singulier "la ténèbre ») ou du traitement de thèmes inconvenants, Millet est donc fort peu classique au sens strict du terme. Mais bien évidemment la coprésence d'éléments sordides d'une part, de marqueurs du registre soutenu et du style élevé d'autre part (le passé simple, la concordance des temps au subjonctif imparfait, les phrases complexes à plusieurs subordonnées), met en avant un e ff e t classique. Pour l'illustrer :

12 R. Millet, L'Amour des trois sours Piale, Paris, Gallimard, 1999, p. 56.

13 « les choses complexes et les sordides doivent être dites en une langue dont la tenue soit à mille lieues de l'abjection et de la noirceur qu'elle dévoile " (R. Millet, Le Goût des femmes laides, Paris, Gallimard, 2005, p. 68).

${ }^{14}$ R. Millet, Lauve le pur, Paris, Gallimard, 2001, p. 32.

15 Ibidem, p. 42. 
Aujourd'hui, elle eût remplacé " emmerdant " par " chiant », [...] ce qui peut faire penser que lorsque le langage fait appel à sa propre sentine, il ne reste plus qu'à oublier la syntaxe pour que la langue soit rendue aux onomatopées, gestes par lesquels on tente de pallier le défaut de langage. ${ }^{16}$

Dans ce passage, le narrateur reprend en mention un mot prononcé par un personnage. Ce phénomène de mention est une des formes que prend, conjointement à ces effets archaïsants et classicisants, la mise à distance de la langue courante moderne. Le phénomène est commun à nos deux auteurs : le rejet de la modernité est aussi rejet des mots qui la dénotent ou la connotent, ce qui conduit Millet comme Bodinat à employer volontairement des termes impropres pour s'y référer. Cela passe, encore une fois, par des archaïsmes (" aéronef $~^{17}$ ) mais surtout des néologismes, comme " rapeux ${ }^{18}$ chez Millet (souligné par l'italique), ou chez Bodinat ces innombrables façons de ne pas nommer comme il se doit les artefacts techniques contemporains, par des néologismes : " tactiphone ${ }^{19}$, des termes sortis de l'usage : " mondovision $»^{20}$, des périphrases : " communicateurs portatifs $»^{21}$, ou par ces caractérisations métonymiques, comme " éclairage atomique ${ }^{22}$. Ce qui est intéressant, c'est qu'on a moins affaire à un rapport mimétique au passé, qu'à un rapport contrarié au présent qui se fige dans une référence au passé. L'usage artificiel du patois par Richard Millet, employé presque systématiquement en mention (ou par d'autres énonciateurs que le narrateur), le prouve assez.

\footnotetext{
${ }^{16}$ R. Millet, Ma vie parmi les ombres, Paris, Gallimard, 2005, p. 458.

17 B. de Bodinat, La vie sur Terre, op. cit., p. 102.

18 R. Millet, Le sentiment de la langue, Petite vermillon, La Table ronde, 2003, p. 254.

${ }^{19}$ B. de Bodinat, Au fond de la couche gazeuse, op. cit., p. 88.

20 Ibidem, p. 108.

21 Ibidem, p. 78.

22 Ibidem, p. 108. Cette pratique se trouve déjà chez Duhamel, lorsqu'il moque la radiophonie et la " téhessef " (G. Duhamel, Scènes de la vie future, Paris, Mercure de France, 1930).
} 


\section{Artifice?}

Tout fait signe, chez ces deux auteurs, vers un répertoire classique, dans le même temps que la pratique stylistique trahit la norme classique stricte. Néanmoins, il y a bel et bien selon moi un horizon classique partagé, une sorte de norme esthétique qui dirige l'écriture, jusque dans ce qu'elle a d'innovant, notamment en termes de grammaire. Chez Millet : "Et pourtant rien ne m'émeut plus que Marina croie encore à autrui ${ }^{23}$. La phrase fusionne le que adverbe comparatif et le qu e complétif, ce qui, au prix d'une audace syntaxique, évite un inélégant "que le fait que ${ }^{24}$. Et chez Bodinat:

Ces questions peuvent préoccuper l'esprit et le distraire en attendant que ce débit incessant des secondes vienne faire buter les aiguilles sur le déclic de l'alarme et de recommencer encore une autre journée dans le tableau horaire, de retourner dans la cage d'écureuil dont ces horloges sont le moyeu. ${ }^{25}$

L'auteur coordonne une complétive et un infinitif précédé de " de ", sur le même plan de subordination, avec, qui plus est, un sujet et un contrôleur différents pour ces deux verbes. Ce qui a pour conséquence de nous faire entendre le second en même temps comme un infinitif de narration : " et de recommencer ", plus propice à suggérer l'aliénation d'un procès où le sujet n'est pas investi. Ailleurs encore, on trouve ce régime étonnant associé à un verbe déjà rare, et d'emploi intransitif : " ces hommes s'invétérant à leur malheur de tenir la femme en sujétion ${ }^{26}$.

${ }^{23}$ R. Millet, Ma vie parmi les ombres, op. cit., p. 38.

24 Millet affiche par ailleurs une certaine nonchalance à l'égard du solécisme quand il préface Saint-Simon, ce qui n'est pas sans contredire ses propos répétés sur l'absence de syntaxe de la littérature contemporaine. Cf. Saint-Simon, Mémoires III : La mort de Louis XIV, Paris, Gallimard, 2007.

${ }^{25}$ B. de Bodinat, Au fond de la couche gazeuse, op. cit., p. 182.

$26 \mathrm{Ibidem}$, p. 109. La seule occurrence d'un emploi non-transitif est répertoriée en 1563 par le TLF : « invétéré en quelque chose “( $d$ 'une personne) 
En somme les solécismes, anacoluthes et innovations grammaticales semblent prendre pied dans un terreau linguistique ancien, et hériter de la souplesse syntaxique du français classique27. Sans réduire leur nouveauté, ces formes sont accompagnées de plusieurs éléments cotextuels qui poussent à sentir en elles une connotation classique. Mais cela dénote aussi une capacité de leur langue à se modifier, à épouser une certaine forme, qui n'est pas préexistante, pour tenir un discours d'un type nouveau.

\section{Figuration}

Pour comprendre cela, il faut s'éloigner de la conception rhétorique du style, qui sépare la forme et le fond, et privilégier une pensée du style comme figuration nécessaire à une pensée ou à un objet nouveaux ${ }^{28}$. Prenons par exemple un trope récurrent chez Millet et Bodinat, l'énumération, qui me semble figurer un rapport au monde, et pas seulement la volonté de créer un effet d'accumulation (grandiloquent ou comique, comme c'est souvent le cas depuis Rabelais) : " dans une Corrèze d'où ces odeurs avaient déjà disparu avec le patois, la tuberculose, les grandes familles, le sens de la syntaxe, et les mystères du christianisme ${ }^{29}$ et :

celui-là ayant connu dans sa jeunesse la mansarde sans eau et les veilles studieuses assiégées par les ombres vivantes de la lampe à pétrole, la ville encore très peuplée de destinée modeste, des chevaux et des char-

endurci par le temps" (Palissy, Recepte véritable, p. 98 ds Hug.) ", cf. http:// www.cnrtl.fr/lexicographie/inv\%C3\%A9terer, consulté le 25 février 2019.

27 Souplesse connue, dans l'usage d'un contrôleur d'infinitif ou de gérondif différant du sujet du verbe principal. Cf. N. Fournier, Grammaire du français classique, Paris, Belin, 1998, p. 294.

${ }_{28}$ Ici, nous pouvons nous reporter à l'article de Laurent Jenny, "Sur le style littéraire ", [dans :] M. Dereu (éd.), Vous avez dit style d'auteur?, Nancy, Presses universitaires de Nancy, 1999. L. Jenny donne pour exemple la phrase proustienne, dont la longueur n'est pas simplement un choix ornemental (phrase longue contre brièveté), mais bien la seule manière possible de figurer et de rendre compte de l'effort mémoriel.

${ }^{29}$ R. Millet, Ma vie parmi les ombres, op. cit., p. 26. 
rettes à bras, d'échoppes, d'escaliers en colimaçon, de vie sociale en famille parmi ces rues de faubourgs d'ancien régime, etc. 30

Dans les deux cas, on assiste au même rapport affectif au passé, dont la cohérence n'est pas démontrée, mais au contraire simplement donnée par l'équivalence présupposée des différents membres de l'énumération, pourtant visiblement hétéroclite. L'énumération aurait donc une fonction argumentative prénotionnelle, trahissant un rapport au passé qui ne peut être rationnel, puisqu'il se fonde précisément sur le rejet de la rationalisation du monde moderne et industrialisé ; et le recours à l'énumération plutôt qu'à d'autres moyens de formuler des présuppositions (enthymèmes, syntagmes associant un nom et une épithète en dissimulant l'acte de prédication, etc.) s'explique par le rapport nécessairement fragmentaire à un passé qui est pour partie méconnu, pour partie disparu ${ }^{31}$.

Cependant on ne peut s'arrêter à ce dénominateur commun que constitue l'énumération hétéroclite. II est visible que leur style diffère, y compris dans la composition des énumérations, et cette différence est à mon avis moins affective que politique. Au tranquille désespoir de Richard Millet s'oppose l'angoisse parfois hystérique de Bodinat, qui dévoile, par son écriture, une préoccupation constante face à la catastrophe du monde industriel, et le désir d'aller malgré tout à l'encontre de l'irrémédiable (dans une perspective qui est bien loin de se limiter à l'écologisme, dont la composante technique est d'ailleurs dénoncée). Une phrase comme : " La machine du progrès

${ }^{30}$ B. de Bodinat, La vie sur Terre, op. cit., p. 163.

31 On pourrait sans doute étendre à d'autres auteurs cette étude de l'énumération comme court-circuit argumentatif et comme lieu de production d'un imaginaire. Chez Debord, on en trouve qui cette fois sont l'expression d'une philosophie de la perte de qualité et donc de l'équivalence des marchandises entre elles : " On peut garder le nom quand la chose a été secrètement changée (de la bière, du bœuf, un philosophe) " (G. Debord, Commentaires sur la société du spectacle suivi de Préface à la quatrième édition italienne de "La Société du Spectacle ", Paris, Gallimard, 1996, p. 42). 
s'est mise en route, et voilà, nous sommes presque arrivés $1{ }^{32}$, utilise deux passés composés. Le premier combine l'aspect accompli et le sens inchoatif du verbe se m e t $\mathrm{t}$ re, le second, l'aspect fini du passé composé semblable à un présent et l'imminence marquée par l'adverbe " presque " - pour faire voir la rapidité historique du processus industriel, la soudaineté d'une catastrophe qui n'a pas eu le temps d'arriver aux consciences. L'identité du temps grammatical écrase deux strates temporelles qu'on voudrait distinguer.

Par ailleurs, il semble possible de dire que Bodinat a un style non-verbal, ou style substantif. II privilégie le groupe nominal au détriment de la proposition subordonnée : " évoquer le musée d'avant la presse des touristes qui font la rentabilité, aux salles paisibles de visiteurs dispersés et murmurants, c'est déjà refuser aux plus nombreux l'horizon culturel élargi ${ }^{33}$; ou " ces accessoires de la vie plus abondée en possibilités ${ }^{34}$; " une thébaïde à l'écart de ces avidités d'abus nerveux, de ces excitations en collectivité de derniers jours ouvrables, de ces dérangements d'esprit dans l'insomnie générale $1^{35}$. Le choix des formes nominales ou adjectives du verbe, ou de substantifs déverbaux (la presse) supprime l'aspect processuel, donne du présent le sentiment d'une perspective bouchée, exclue du temps. La place de l'échappée est restreinte, voire quasi-inexistante chez Bodinat (alors même qu'elle peut prendre des formes diverses dans la littérature anti-industrielle). II y a aussi quelque chose de latin dans ces formules, mais qui précisément génère un étrangement du propos et un figement temporel suscitant l'angoisse à la lecture : tous sentiments qui selon moi s'expliquent par le refus politique, en dernière instance, de renoncer à l'action et d'accepter l'existant comme norme.

32 B. de Bodinat, La vie sur Terre, op. cit., p. 20.

${ }^{33}$ B. de Bodinat, Au fond de la couche gazeuse, op. cit., p. 109.

34 Ibidem, p. 79.

35 Ibidem, p. 119. 
Si l'on entrevoit un style commun que j'ai appelé passéiste, il y a néanmoins entre les deux auteurs une divergence stylistique qu'il faudrait sans doute examiner suivant des catégories politiques.

\section{Conclusion}

Définir un style passéiste comme style " à la manière de " textes du passé permet de mettre en avant ce qui, chez les auteurs que nous avons étudiés, signale (au sens sémiotique) une attitude philosophique et politique, pose un certain horizon d'attente. C'est peut-être d'abord de là qu'il faudrait partir si l'on s'intéressait au caractère social du style. Mais pour caractériser une pensée dans sa dimension affective (que celle-ci soit individuelle ou collective d'ailleurs), il est nécessaire de ne plus considérer le style simplement comme choix ornemental s'accordant à une pensée, mais bien comme expression nécessaire d'une certaine vision qui ne peut pas se rapporter autrement au référent en question. En ce sens, le style est la forme littéraire que prend un certain rapport à l'époque. 


\section{bibliographie}

Antoine G., Les Cinq Grandes Odes de Claudel ou La poésie de la répétition, Paris, Minard, 1959.

Antoine G., La coordination en français, Paris, Éditions D’Artrey, 1958.

Bodinat B. de, La vie sur Terre : réflexions sur le peu d'avenir que contient le temps où nous sommes, Paris, Éditions de l'Encyclopédie des Nuisances, 2008.

Bodinat B. de, Au fond de la couche gazeuse : 2011-2015, Paris, Fario, 2015. Compagnon A., Les Antimodernes : de Joseph de Maistre à Roland Barthes, Paris, Gallimard, 2005.

Debord G., Commentaires sur la société du spectacle suivi de Préface à la quatrième édition italienne de "La Société du Spectacle ", Paris, Gallimard, 1996.

Duhamel G., Scènes de la vie future, Paris, Mercure de France, 1930.

Fournier N., Grammaire du français classique, Paris, Belin, 1998.

Himy-Piéri L., et Macé S. (éd.), Stylistique de l'archaïsme: Colloque de Cerisy, Bordeaux, Presses universitaires de Bordeaux, 2010.

Jenny L., "Sur le style littéraire ", [dans :] M. Dereu (éd.), Vous avez dit style d'auteur?, Nancy, Presses universitaires de Nancy, 1999.

Lapaque S., Autrement et encore, Paris, Actes Sud, 2013.

Le Trésor de la Langue Française, http://www.cnrtl.fr/lexicographie/inv\% C3\%A9terer, consulté le 25 février 2019.

Millet R., L'Amour des trois sœurs Piale, Paris, Gallimard, 1999.

Millet R., Lauve le pur, Paris, Gallimard, 2001.

Millet R., Le sentiment de la langue, Petite vermillon, La Table ronde, 2003.

Millet R., Ma vie parmi les ombres, Paris, Gallimard, 2005.

Millet R., Le Goût des femmes laides, Paris, Gallimard, 2005.

Millet R., Tarnac, Paris, Gallimard, 2010.

Plazenet L., " Voix de l'ombre et du néant », [dans :] J.-Y. Laurichesse (éd.), Littératures, Toulouse, Presses universitaires du Mirail, 2011, vol. 63.

Rimbaud A., Une saison en enfer, Alliance typographique, Bruxelles, 1873. Saint-Simon, Mémoires III : La mort de Louis XIV, Paris, Gallimard, 2007.

\section{abstract}

\section{Writing backwards: can stylistics be applied to passeism?}

This article intends to examine the question of return in literature by using the tools of stylistics. It also seeks to question the relevance of those tools for this very object, in which individual and collective issues overlap. We chose to look at two 'past-styled authors', Richard Millet and Baudouin de Bodinat. Their writings allow us to grasp the connection between style and ideology, but also to compare different stylistic methods (mainly a rhetorical approach or a figurative conception of style) pointing to different conclusions. 


\section{keywords}

style, antimodernism, Millet, Bodinat

\section{mots-clés}

style, antimodernes, Millet, Bodinat

\section{vincent berthelier}

Vincent Berthelier est en thèse à la Sorbonne sous la direction de Christelle Reggiani et de Gilles Philippe. Ses recherches portent sur la droite littéraire du XXe siècle. II coorganise le séminaire "Lectures de Marx " et le "Séminaire Littéraire des Armes de la Critique " (SLAC) à l'École Normale Supérieure de la rue d'Ulm, et participe à la traduction française des œuvres complètes de Rosa Luxemburg.

ORCID : http://orcid.org/0000-0003-0156-2390 